\title{
Genetic engineering of stem cells by non-viral vectors
}

\author{
Catarina Madeira $^{1,2, *}$, Sofia C. Ribeiro ${ }^{2}$, Rui Mendes $^{2}$, Irina S.M. Pinheiro ${ }^{2}$, Cláudia L. da Silva ${ }^{1,2}$, Joaquim M.S. Cabral ${ }^{1,2}$ \\ ${ }^{1}$ Department of Bioengineering, Instituto Superior Técnico (IST) \\ ${ }^{2}$ Institute for Biotechnology and Bioengineering (IBB), Centre for Biological and Chemical Engineering (CEBQ), IST \\ Lisboa, Portugal \\ *catarina.madeira@ist.utl.pt
}

\begin{abstract}
Stem/progenitor cells hold a great promise for application in several therapies due to their unique biological characteristics. With the purpose of harnessing these cells full potential in cell-or gene-based therapies it might be advantageous to enhance some of their features through gene delivery strategies. Accordingly, we are interested in developing efficient and safe methodologies to genetically engineer stem cells, boosting their therapeutic efficacy in Regenerative Medicine. In our work, delivery of plasmid DNA to human Bone Marrow Mesenchymal Stem Cells (BM-MSC) was optimized by lipofection and by a recently available microporation technique and no effect was observed in their immunophenotypic characteristics or differentiative potential. After lipofection similar number of plasmid copies was determined at different cell passages. Importantly, cell proliferation kinetics slowed down due to the presence of plasmid. Overall, we believe our findings are extremely useful towards the maximization of gene delivery to human MSC, without compromising cell function and viability.
\end{abstract}

Index Terms - gene delivery, lipofection, microporation, plasmid DNA, stem cells

\section{CONTEXT}

Stem cells own high potential of self-renewal, proliferation and differentiation into different cell lineages therefore being considered outstanding candidates to cure large number of diseases or tissue injuries.

Genetically engineering of stem/progenitors cells is extremely advantageous both in basic research and clinical settings since it allows boosting their ex-vivo proliferation or differentiation towards a specific phenotype (Fig. 1). Additionally, the overexpression of homing-related receptors or other specific proteins may also increase their capacity to specifically target damaged tissues and deliver therapeutic proteins.

Gene delivery to stem cells has been frequently accomplished by viral-based vectors, but some issues regarding these vectors safety and manufacturing have encouraged the development and optimisation of non-viral based techniques. Indeed, naked or complexed plasmid DNA is safer and has no limitation in the molecular size of DNA applied. As these vectors have not yet achieved the gene transfer efficiency of its viral counterparts, the design and analysis of new polymers, lipids, peptides, and physical methods that can efficiently deliver DNA in vivo and/or exvivo is continuously under study (see review [1]). Moreover, and to our best knowledge no reports have focused on the effect of plasmid DNA on stem cells proliferation kinetics and few studies have assessed the efficiency of gene delivery at different cell passages.

Figure 1. Schematic illustration of potential future ex-vivo manipulation of stem/progenitor cells. After being isolated, adult stem cells will be transfected with a plasmid encoding a therapeutic protein. Depending on the applications, cells might be expanded and/or differentiated and further infused or implanted into the patient.

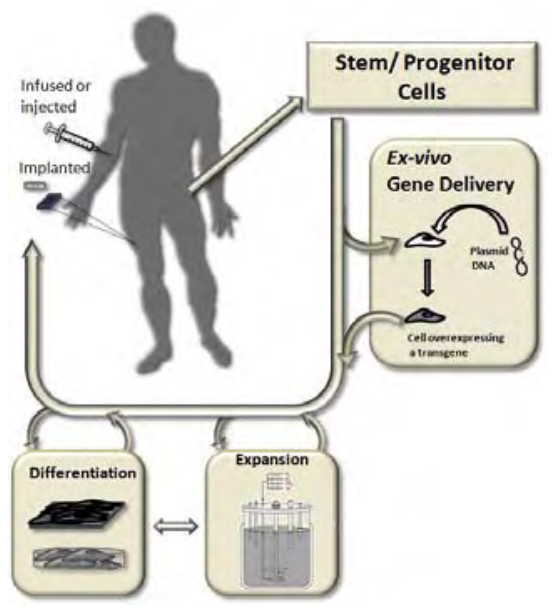

In particular, MSC have been shown to be exceptionally promising for ex vivo therapy in regenerative medicine and cancer treatment applications, which will certainly be expanded as soon as suitable non-viral gene transfer protocols are established. Taken together, our results will certainly be very useful in gene delivery applications to stem cells.

\section{GOALS}

Our work is focused on the development of efficient and safe non-viral gene delivery strategies to transiently engineer stem cells. Thus far, we successfully delivered plasmid DNA to BM-MSC by cationic liposomes (Lipofection) and a recently available microporation technique.

The approach we have been used in this study aims to investigate the effect of several parameters on the capability of $\mathrm{BM}-\mathrm{MSC}$ to receive foreigner DNA, such as cell passage, and

\footnotetext{
$1^{\text {st }}$ Portuguese Meeting in Bioengineering, February 2011 Portuguese chapter of IEEE EMBS

Instituto Superior Técnico, Technical University of Lisbon
} 
also to assess the proliferative kinetics, immunophenotypic characteristics and differentiative potential of these cells transiently expressing a reporter gene.

The ultimate goal of the project is to improve stem cells exvivo expansion or their controlled differentiation by overexpressing stemness- or specific lineage commitment related genes/proteins, respectively (Fig. 1). Eventually, the genetically modified cells might be applied to regenerative medicine and tissue engineering applications.

\section{TEAM AND INSTITUTIONS}

This project has been developed under the framework of the Stem Cell Bioengineering Laboratory (SCBL) at the Institute for Biotechnology and Bioengineering at Instituto Superior Técnico under the supervision of the team leader Professor Joaquim Sampaio Cabral.

\section{IMPLEMENTATION}

BM-MSC used in these studies, were obtained from volunteer donors at Instituto Português de Oncologia (IPO) de Lisboa Francisco Gentil after informed consent. Low-density BM mononuclear cells were separated by a Ficoll density gradient (1.077 g/mL) (GE Healthcare, Uppsala) and MSC were isolated based on their adherence to plastic and further cultured, expanded and characterized as previously described $[2,3]$.

Two different methodologies were used to deliver genes to MSC: lipofection, using Lipofectamine 2000 (Invitrogen) and microporation, using a Microporator MP100/Neon (Invitrogen). Both methods were carried out according to supplier instructions and further details are given elsewhere [2, 3]. Twenty four hours after delivering plasmid DNA encoding green fluorescent protein (pVAX-GFP; $3697 \mathrm{bp}$ ), the percentage of transfected cells, the level of protein expression, immunophenotypic characteristics and cells proliferative kinetics were analysed by flow cytometry (FACScalibur equipment/CellQuest software (BD Biosciences)) [2]. The percentage of viable cells (cell viability) was estimated by trypan blue exclusion method. For each micro-electroporated sample $(\mathrm{m})$, cell recovery $(\mathrm{CR})$ was determined using the equation $\mathrm{CR}(\%)_{\mathrm{m}}=\left(\mathrm{CA}_{\mathrm{m}} / \mathrm{CA}_{\mathrm{c}}\right) \times 100$, where $\mathrm{CA}$ is the number of cells alive and ${ }_{c}$ is the non-electroporated control. Yield of transfection (Y) was calculated using the equation $\mathrm{Y}$ $(\%)_{\mathrm{m}}=\left(\mathrm{GFP}^{+} \times \mathrm{CA}_{\mathrm{m}}\right) / \mathrm{CT}_{\mathrm{c}}$ where $\mathrm{CT}$ is the number of total cells.

The number of plasmid copy number inside the cells after gene delivery was determined by RT-PCR [4]. When transfecetd MSC reached total confluence, osteogenic and adipogenic differentiation was induced through replacement of expansion medium by specific differentiation medium. After two weeks cells were stained as previously described [3].

\section{RESULTS}

\section{A. Influence of Cell Passage Number on Transfection Efficiency.}

Typically, as MSCs are rare in BM niches, they have to be cultivated for several passages in order to reach clinically relevant cell numbers. Thus, the influence of MSC passage number on transfection efficiency was studied herein. To this end, MSCs from 2 different donors, from passage 1 to 4, were used. The cells were analysed by evaluating the number of cells expressing GFP (data not shown), the MFI value (measure of GFP expression level) (Fig. 2A), and the number of plasmids per cell (Fig. 2B).

Figure 2. Mean fluorescence intensity assessed by flow cytometry analysis (A) and intracellular plasmid copy number determined by RT-PCR (B) after lipofection of MSC (Lipid/DNA $(\mu \mathrm{L} / \mu \mathrm{g})=1.25$ ) of two different donors, at different cell passages [3].
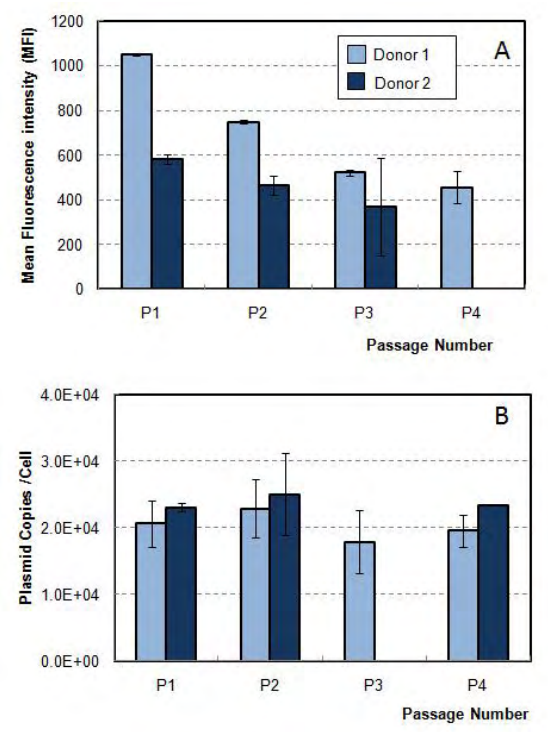

Indeed, the quantification of plasmid DNA (pDNA) molecules that enter the cells assisted by cationic liposomes can not only contribute to understand the underlying mechanisms of liposomal gene delivery but it also can be a helpful tool to optimize the transfection efficiencies of liposomal vectors. Accordingly, we were able to quantify by Real Time-PCR the optimal amount of delivered DNA required for the best transgene expression [4] and further compared the delivery efficiency to human MSC at different cell passages. We verified that human MSC transgene expression is cell passage dependent and regardless the decrease in number of transfected cells or level of transgene expression along cell passages, the same amount of plasmid (20,000 pDNA copies/cell) entered the cells (Fig. 2B). We hypothesize that upon the first passages the transcriptional mechanism of MSC is being delayed which may explain the decrease of protein expression whereas cells enclose similar amounts of plasmid DNA.

This perception will undoubtedly be extremely useful in future developments of novel vectors for nonviral gene 
delivery to human MSC, contributing to an optimization of the gene delivery processes as well as to broadening the field of MSC applications.

\section{B. Effect of DNA amount on MSC microporation}

We analyzed the effect of DNA amount on gene delivery efficiency to MSC by microporation using one pulse of $1000 \mathrm{~V}$ during $40 \mathrm{~ms}$. It appears that the increase of DNA amount from 1 to $3 \mu \mathrm{g}$ did not significantly affect the number of transfected cells $(p=0.17)$, slightly decreased cell viability $(p$ $=0.018)$ and considerably decreased cell recovery $(p=0.004)$ (Fig. 3A).

Figure 3. Microporation of BM-MSC using $1000 \mathrm{~V}, 40 \mathrm{~ms}$ and one pulse (A) Percentage of $\mathrm{GFP}^{+}$cells, cell viability, cell recovery and yield of transfection using $1 \mu \mathrm{g}$ (white bar) and $3 \mu \mathrm{g}$ (grey bar) of pDNA. Results were obtained from two independent experiments with duplicates $( \pm$ S.E.M.). Statistical differences are indicated with $*$ for $p<0.05$ [2]. (B) Percentage of $\mathrm{GFP}+$ cells and mean fluorescence intensity (MFI) decay along 25 days. (C) Microscope fluorescence image of MSC 24h after microporation.
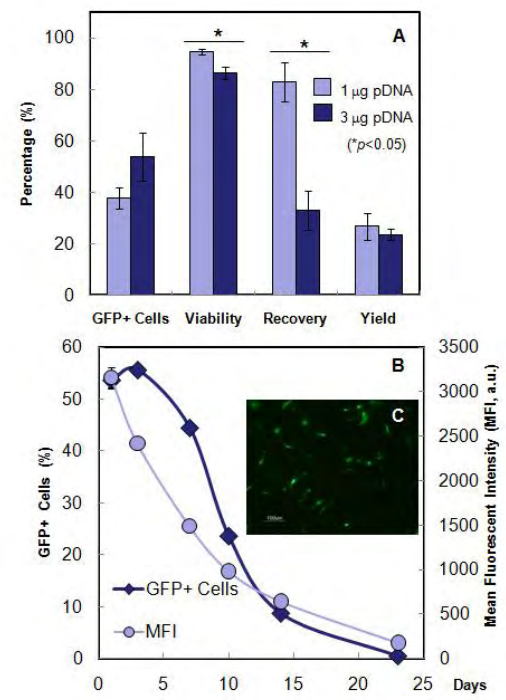

We consider that the best results were achieved with $1 \mu \mathrm{g}$ of pDNA, because $85 \%$ of cells were recovered concomitantly with $40 \%$ of transfected cells leading to an overall yield of transfection of $35 \%$ which is the highest value, reported so far. For most applications in the stem cell field (from basic research to clinic use) the avoidance of cell death is usually a concern [5]. In fact, for specific applications such as wound healing, a lower expression of protein within high number of cells might be more appropriated than high levels of expression in lower cell number.

Recently, Wang et al. reported that microporation of adipose derived MSC using 1.5-2 $\mu \mathrm{g}$ of plasmid DNA lead to $65 \%$ of $\mathrm{GFP}^{+}$cells with only $15 \%$ of cell toxicity measured by Lactate dehydrogenase assay [6]. In addition, other authors have used the same technique, with similar parameters, to transfect umbilical cord blood-derived MSC and obtained nearly $80 \%$ of transfected cells with $80 \%$ of cell survival [7].
These authors also compared microporation with other transfection methods and verified that this method was more efficient and caused minimal cell damage.

As detailed in Fig 3B, we were able to observe MSC expressing the reporter protein throughout three weeks. In addition, cells expressing the transgene (Fig. 2C) maintained the characteristic spindle-shaped morphology of non-modified MSC.

\section{Cell Division Kinetics after gene delivery}

An existing protocol that uses a PKH26 dye (Sigma) was adapted to be used within microporation of cells. From data shown in Fig. 4A we can verify that after 4 days in culture, most non-electroporated cells belong to generation 3 while on day 7 most cells are in generation 4; interestingly, some actively dividing cells have reached generation 8 after this time period.

Figure 4. Number of doubling generations of MSC in culture along 7 days without being submitted to microporation (A), and microporated with pDNA (B). Data were acquired by flow cytometry using cells previously stained with PKH26 membrane dye. Each bar represents the percentage of cells in each doubling generation (Generations1-8), that MSC have undergone at each point in culture [2]

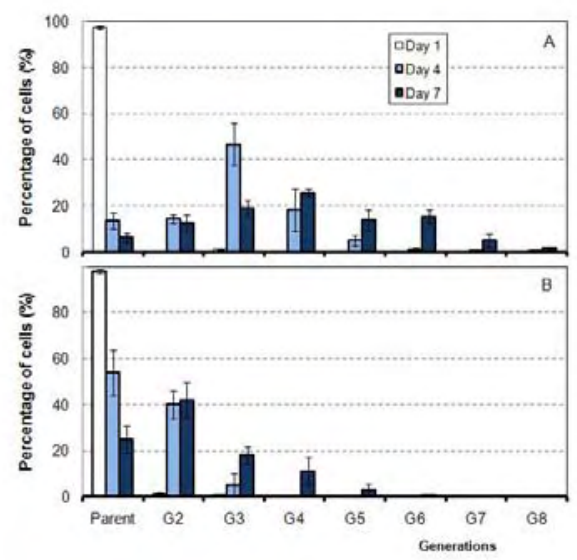

Similar results were obtained after microporating cells in the absence of pDNA. On the other hand, the presence of pDNA inside the cells was shown to delay cell division kinetics because on day 4 most cells remained in the first generation (Fig. 4B). On day 7, most cells were still on generation 2 which largely differs from the results obtained without pDNA. It may be reasonable to assume that microporation by itself does not diminish cell division kinetics but the presence of pDNA clearly delays the cell division. The presence of $\mathrm{CpG}$ motifs in the plasmid backbone might be the responsible for these effects and new plasmids lacking these motifs are currently being used by us. 


\section{Cell Effect of microporation on BM-MSC multipotency}

After microporation, MSC were tested for their characteristic immunophenotype and it was verified whether transfected MSC maintained their characteristic phenotypic profile, namely over $90 \%$ positive for CD73 and CD105.

Figure 5. Immunophenotype evaluation of non-microporated cells (A), microporated cells without pDNA (B) and in the presence of $3 \mu \mathrm{g}$ of pDNA (C), $24 \mathrm{~h}$ after microporation using $1400 \mathrm{~V}, 30 \mathrm{~ms}$ in a sacarose based buffer (Ref JB). Alkaline Phosphatase (D) and Oil red-O (E) stainings were tested in order to analyse the maintenance of osteogenic and adipogenic differentiation potential after transfection, respectively [2].
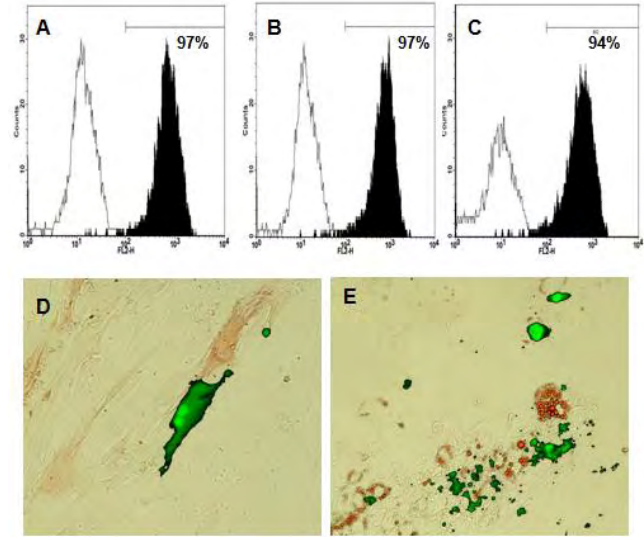

More than $90 \%$ of cells (non microporated and microporated without pDNA) expressed these markers since the day after microporation (Fig. 5A and B) with the exception of transfected cells. Although the receptor CD73 did not seem to be affected in transfected cells $(93.3 \pm 1.4 \%$ ) (Fig. 5C), a slight lower percentage of cells were expressing the surface marker CD105 $(84.5 \pm 0.7 \%)$. Nonetheless, four days after microporation $93 \%$ of transfected cells were expressing CD105 surface receptors (data not shown). As can be depicted from Fig. 5D and E no effect was observed on osteogenic or adipogenic differentiative potential of microporated BM-MSC in the presence of pDNA. Noteworthy is that after two weeks of differentiation protocols, few cells expressing GFP were still visible under the fluorescence microscope.

\section{CONCLUSIONS}

In this work we established two different methods for nonviral gene delivery into human BM-MSC: lipofection and microporation.

We verified that cationic liposomes are promising transfection agents to human MSC, especially for applications only requiring low and transient expression. Furthermore, we found that human MSC lipofected at different passages, using the same amount of plasmid displayed different levels of protein expression regardless similar amount of plasmids were detected inside the cells.
We also emphasize the relationship that may be established between high levels of transgene expression and cell mortality that in most cases seems to be related to the presence of plasmid inside the cells and not so much with the gene delivery process itself. By achieving high yields of transfection with human adult MSC, known as hard-to transfect cells, microporation may also be extremely useful for generation of induced pluripotent cells where high cell recoveries and high gene expression are crucial.

The establishment of the aforementioned methodologies and the achieved results offers a valuable insight in the field of stem cells bioengineering which undoubtedly will pave the way to consider non-viral gene delivery methodologies as essential tools in future clinical applications.

\section{PLANNED DEVELOPMENTS}

On-going work encompasses the use of novel plasmids lacking $\mathrm{CpG}$ motifs in order to assess proliferative kinetics of stem cells. In a short term, the reporter gene will be replaced by a therapeutic gene involved in specific differentiation pathways.

\section{ACKNOWLEDGMENT}

The authors acknowledge funding from the MIT-Portugal Program, Bioengineering Focus Area, Fundação para a Ciência e Tecnologia and from Associação Portuguesa contra a Leucemia (Portuguese Association against Leukemia).

\section{REFERENCES}

[1] D. Sheyn, O. Mizrahi, S. Benjamin, Z. Gazit, G. Pelled, D. Gazit, "Genetically modified cells in regenerative medicine and tissue engineering" Advanced Drug Delivery Reviews, Vol. 62, pp. 683-698, 2010 .

[2] C. Madeira, S.C. Ribeiro, I.S.M. Pinheiro, S.M.A. Martins, P.Z. Andrade, C.L. da Silva, J.M.S. Cabral "Gene Delivery to human bone marrow mesenchymal stem cells by microporation" Journal of Biotechnology, Vol. 151, pp. 130-136, 2011.

[3] C. Madeira, R.D. Mendes, S.C. Ribeiro, J.S. Boura, M.R. Aires-Barros, C.L. da Silva, J.M.S. Cabral "Nonviral Gene Delivery to Mesenchymal Stem Cells Using Cationic Liposomes for Gene and Cell Therapy". Journal of Biomedicine and Biotechnology, Doi: 10.1155/2010/735349.

[4] S.C. Ribeiro, R.D. Mendes, C. Madeira, G.A. Monteiro, C.L. da Silva, J.M.S. Cabral "A Quantitative Method to Evaluate Mesenchymal Stem Cell Lipofection Using Real-Time PCR" Biotechnology Progress, Vol. 26, pp. 1501-1504, 2010.

[5] C. Madeira, S.C. Ribeiro, J.S. Boura, C.L. da Silva, J.M.S. Cabral "Microporation of human mesenchymal stem cells promotes high cellular recoveries and efficient plasmid gene delivery" Human Gene Therapy, Vol. 20 , pp. 1490-1491, 2009.

[6] Y.H. Wang, M.L. Ho, J.K. Chang, H.C. Chu, S.C. Lai, G.J. Wang, "Microporation Is a Valuable Transfection Method for Gene Expression in Human Adipose Tissue-derived Stem Cells" Molecular Therapy, Vol. 17, pp. 302-308, 2009.

[7] J.Y. Lim, et al. "Microporation is a valuable transfection method for efficient gene delivery into human umbilical cord blood-derived mesenchymal stem cells" BMC Biotechnology, Vol. 10, 2010. 